\title{
Incorporações de medicamentos para doenças raras no Brasil: é possível acesso integral a estes pacientes?
}

\author{
Incorporation of drugs for rare diseases in Brazil: \\ is it possible to have full access to these patients?
}

Luiza Vasconcelos Biglia (https://orcid.org/0000-0003-1403-4167) ${ }^{1}$

Samara Jamile Mendes (https://orcid.org/0000-0003-3107-8233) ${ }^{1}$

Tácio de Mendonça Lima (https://orcid.org/0000-0003-4395-2098) ${ }^{2}$

Patricia Melo Aguiar (https://orcid.org/0000-0002-3957-4533) ${ }^{1}$

${ }^{1}$ Faculdade de Ciências Farmacêuticas, Universidade de São Paulo. Av. Prof. Lineu Prestes 580 Conj. das Químicas Bloco 13, Cidade Universitária, Butantã. 05508-900 São Paulo SP Brasil. aguiar.pm@usp.br ${ }^{2}$ Departamento de Ciências Farmacêuticas, Universidade Federal Rural do Rio de Janeiro. Seropédia RJ Brasil.
Abstract This study aims to describe the profile for the requested incorporation of rare disease drugs submitted to CONITEC and its recommendations, comparing the incorporation criteria employed by other HTA agencies globally. To this end, requests for the treatment of rare diseases submitted to CONITEC from July 2012 to June 2019 and its recommendations to the Brazilian Unified Health System (SUS) were included in this study. Subsequently, we compared the criteria used by CONITEC and other HTA agencies to incorporate these drugs. Sixty medicine incorporation requests to treat thirty rare diseases were submitted to CONITEC. Pharmaceutical companies made the most requests (66\%). Budget impact analyses were presented in $85 \%$ of the requests and HT economic analyses in $68 \%$. A total of $52 \%$ of the requests were incorporated into the SUS. CONITEC's justifications for the non-incorporation were the lack of quality clinical evidence, non-cost-effective technologies, and modest clinical benefits that do not justify the high prices. International HTA agencies (CAN, UK, FR, AUS) use different criteria for rare diseases assessments. The data indicate that most of the evaluated drugs were incorporated into the SUS, and adopting different criteria to assess the incorporation of rare diseases medicines will possibly strengthen decision-making.

Key words Rare diseases, Health technology assessment, Unified Health System
Resumo Descrever o perfil de solicitações de incorporação de medicamentos para doenças raras (DR) enviadas à Comissão Nacional de Incorporação de Tecnologias no SUS (CONITEC) e suas recomendações, comparando critérios usados para incorporação com outras agências de avaliações de tecnologias em saúde (ATS) no mundo. Para tanto, foram avaliadas as solicitações submetidas à CONITEC e suas recomendações ao SUS, de julho de 2012 a junho de 2019, para tratamento de DR. A seguir, foi feita comparação dos critérios utilizados pela CONITEC e por outras agências de ATS para incorporação destes medicamentos. Houve 60 solicitações de incorporação para 30 DR à CONITEC. A maioria das solicitações (66\%) foi feita por indústrias farmacêuticas. Análises de impacto orçamentário foram apresentadas em 85\% das solicitações e análises econômicas de tecnologias em saúde em 68\% delas. Trinta e duas (52\%) do total de avaliações foram incorporadas ao SUS. As justificativas da CONITEC para a não incorporação foram ausência de evidência clínica, tecnologias não custo-efetivas e modestos benefícios clínicos que não justificam o preço. Agências de ATS internacionais (UK, FR, CAN, AUS) usam critérios diferenciados para avaliações de DR. Os dados apontam que a maioria dos medicamentos avaliados foi incorporada ao SUS e que a adoção de critérios diferenciados para avaliação da incorporação de medicamentos para $D R$ possivelmente trará robustez à tomada de decisão. Palavras-chave Doenças raras, Avaliação da tecnologia em saúde, Sistema Único de Saúde. 


\section{Introdução}

No âmbito da saúde pública no Brasil, foi criada em 1990 a Lei 8.080 que constituiu o Sistema Único de Saúde (SUS) que tem como princípios a Universalidade, a Integralidade e a Equidade ${ }^{1}$, respeitando o Art. 196 da Constituição de 1988 a saúde é um direito de todos e dever do Estado ${ }^{2}$. $O$ princípio da Universalidade preconiza o acesso à saúde a todos os cidadãos. A Equidade permitiria a ausência de diferenças injustas, evitáveis ou remediáveis na saúde de populações ou grupos definidos com critérios sociais, econômicos, demográficos ou geográficos ${ }^{3}$. Já o conceito de Integralidade, mais especificamente, apresentase como a necessidade de compreender o indivíduo enquanto ser biopsicossocial em sua essência, passando pela possibilidade de acesso a todos os níveis do sistema, o que nos leva a sua prática mais concreta, com a intenção de dar-lhe materialidade à vida dos serviços ${ }^{4}$. Sendo assim, Carnut ${ }^{4}$ definiu a integralidade como um princípio-diretriz do SUS. Princípio, pois, se vale da explanação filosófica do ser humano, e diretriz por ter se tornado algo concreto, que guia o processo de trabalho.

Neste contexto, o acesso específico a medicamentos é assegurado por duas políticas públicas de saúde, a Política Nacional de Medicamentos ${ }^{5}$ e a Política Nacional de Assistência Farmacêutica ${ }^{6}$. Contudo, nos últimos anos, o SUS tem apresentado alguns desafios para a sua sustentabilidade, entre eles, assegurar o seu financiamento. A pesquisa científica na área da saúde tem produzido aumento expressivo de alternativas de detecção, prevenção e tratamento de doenças, porém restrições orçamentárias não permitem que os sistemas de cuidado à saúde ofereçam todas as intervenções para todos os pacientes ${ }^{7}$.

No campo de avaliações de tecnologias em saúde (ATS) e, frente à necessidade crescente de eficiência na alocação dos recursos em saúde, sentiu-se, então, a necessidade da criação de uma comissão para auxiliar o Ministério da Saúde na tomada de decisão, chamada de Comissão Nacional de Incorporação de Tecnologias no SUS (CONITEC). Esta comissão foi instituída em 28 de abril de 2011, data em que foi publicada a lei $\mathrm{n}^{\circ} 12.401$, sendo definidos critérios e prazos para a incorporação de tecnologias no SUS ${ }^{8}$.

É um grande desafio da área de ATS a garantia de que também sejam realizadas análises, registros e disponibilização de medicamentos para doenças raras (DR) $)^{9}$. O termo coletivo - doenças raras - é usado para incluir um grupo muito he- terogêneo de distúrbios que pode afetar qualquer sistema do organismo. Também são chamadas de doenças "órfãs", pois, usualmente, se conhece pouco sobre as suas causas e terapias eficazes ainda são limitadas ${ }^{10}$. A maioria destas doenças é de origem genética, muitas vezes incapacitante, que afeta substancialmente a expectativa de vida e prejudica as habilidades físicas e mentais, reduzindo a qualidade de vida do indivíduo. Estas doenças são consideradas um importante problema de saúde pública, sendo negligenciadas por muitos anos pelos sistemas de saúde e indústrias farmacêuticas ${ }^{11}$.

A Organização Mundial de Saúde, em 2012, mapeou entre cinco mil e oito mil doenças raras. $\mathrm{O}$ conceito de doenças raras adotado por este grupo envolve características da doença e fatores epidemiológicos, com determinação de baixa prevalência ${ }^{12}$. É importante notar que a definição epidemiológica varia entre diferentes países. No Brasil, por exemplo, em 2014, o Ministério da Saúde estabeleceu como doença rara aquela que possui prevalência abaixo de 65 a cada cem pessoas ${ }^{13}$. A real prevalência destas doenças é difícil de estimar, já que a literatura tem apontado a ausência de dados confiáveis, baixa consistência entre fontes de informação e baixa qualidade metodológica dos estudos epidemiológicos ${ }^{14}$.

Após ampla discussão com agentes do governo, pesquisadores, médicos e associações de pacientes, foi promulgada em 2014 a Política Nacional de Atenção Integral às Pessoas com Doenças Raras no SUS ${ }^{13}$, a qual busca atenção mais digna, humana e inclusiva para aqueles com doenças raras. Esta Política apresenta como um dos princípios norteadores a incorporação de medicamentos voltados para as doenças raras e indicados no âmbito do SUS, que devem ser resultado das recomendações formuladas por órgãos governamentais a partir do processo de avaliação e aprovação pela CONITEC. Partindo desse princípio e para garantir a efetivação dos objetivos desta Política, a primeira ação desempenhada pelo Ministério da Saúde foi um painel de especialistas que priorizou doze protocolos clínicos para a atenção integral às pessoas com doenças raras ${ }^{15}$.

Apesar dos avanços nos últimos anos, o cumprimento dos princípios da universalidade, da equidade e de integralidade no SUS ainda é um desafio à luz da Política Nacional de Atenção Integral às Pessoas com Doenças Raras ${ }^{16}$. A escassez de evidências científicas adequadas, junto com o alto custo dos tratamentos, acaba por dificultar a incorporação destas terapias e, muitas vezes, 
ocorre transferência à Justiça a decisão sobre o acesso à produtos de saúde pelo indivíduo ${ }^{17,18}$. Diante deste cenário, as doenças raras têm demandado atenção de pesquisadores e de tomadores de decisão, no sentido de verificar se estas devem ganhar critérios de avaliação diferenciados em comparação às demais doenças no âmbito da ATS 9 . Sabendo que pacientes acometidos com alguma doença rara representam uma importante parcela da população, torna-se essencial entender os resultados do processo de incorporação das tecnologias disponibilizadas para estes, comparando com países que também possuem agências de ATS consolidadas.

O presente estudo tem como objetivo descrever o perfil de solicitações de incorporação de medicamentos para doenças raras enviadas à CONITEC e suas recomendações, comparando os critérios usados para incorporação de medicamentos para doenças raras com outras agências de ATS reconhecidas mundialmente.

\section{Métodos}

\section{Perfil das solicitações de incorporação de medicamentos para doenças raras no Brasil}

Trata-se de estudo descritivo e exploratório realizado por meio de levantamentos e análises quantitativas das solicitações de incorporação de medicamentos enviadas à CONITEC e suas recomendações ao SUS, no período de julho de 2012 - mês e ano de início das publicações das avaliações no site online (http://conitec.gov.br/) - a junho de 2019 para tratamento de doenças raras.

Os dados referentes às submissões e às recomendações da CONITEC foram coletados de documentos disponibilizados em seu site online (http://conitec.gov.br/), considerando o critério estabelecido pela Portaria no 199 , de 30 de janeiro de 2014, chamada de Política Nacional de Atenção Integral às Pessoas com Doenças Raras, em que se considera como doença rara aquela que afeta até 65 pessoas em cada cem mil indivíduos ${ }^{13}$.

A análise quantitativa das recomendações positivas ou negativas foi realizada após a seleção de todas as solicitações que se enquadraram na definição mencionada anteriormente de doença rara. A seguir, as solicitações foram apresentadas quanto às indicações dos medicamentos, princípios ativos, demandante, ano, tipo de estudo para evidência clínica, tipo de avaliação econômica, recomendação inicial, recomendação pós consulta pública e resultado da incorporação requerida.
Os dados coletados foram armazenados e analisados em planilha pré-formatada do Microsoft Office Excel ${ }^{\circledR} 365$ ProPlus. Uma estatística descritiva foi utilizada e os resultados foram expressos como frequência absoluta e/ou relativa.

\section{Critérios utilizados por outras agências de ATS para incorporação de medicamentos para doenças raras}

Foi realizada análise comparativa dos critérios usados pela CONITEC para incorporação de medicamentos para doenças raras no Brasil e por agências de ATS internacionais. As agências de ATS de interesse foram escolhidas devido ao pioneirismo na área e por pertencerem a países cujo sistema de saúde se assemelha ao brasileiro. São elas: Pharmaceutical Benefits Advisory Committee (PBAC) da Austrália, Canadian Agency for Drugs and Technologies in Health (CADTH) do Canadá, The National Institute for Health and Care Excellence (NICE) do Reino Unido e Haute Autorité de Santé (HAS) da França. Para tanto, realizou-se pesquisa bibliográfica nas bases de dados PubMed e SciELO, considerando período de busca até junho de 2019, além de consulta aos web sites das referidas agências de ATS.

\section{Resultados}

\section{Análise geral das solicitações de incorporação}

Entre os meses de julho de 2012 a junho de 2019 houve 60 solicitações de incorporação de medicamentos para o tratamento de 30 doenças raras à CONITEC (Quadros 1 e 2). Vale ressaltar que, em 2018, um demandante fez uma submissão de incorporação para tratamento de Uveítes, entretanto, a CONITEC, durante sua avaliação, deliberou dois subgrupos da doença, Uveítes Ativas e Uveítes Inativas. Por esse motivo, analisaremos 60 solicitações e 61 recomendações.

A maioria das solicitações de incorporação enviadas à CONITEC foi realizada pela indústria farmacêutica $(40 ; 66 \%)$, seguido pela Secretaria de Ciência, Tecnologia e Insumos Estratégicos do Ministério da Saúde - SCTIE/MS (11; 18\%) e Secretaria de Atenção à Saúde do Ministério da Saúde - SAS/MS (6;10\%). Apenas uma pequena parcela foi solicitada por governos estaduais (2; 3\%), poder judiciário $(1 ; 2 \%)$ e ambos indústria e médico juntos $(1 ; 2 \%)$. As solicitações foram realizadas com base em níveis variados de 


\begin{tabular}{|c|c|c|c|c|c|c|c|c|c|c|c|c|c|}
\hline 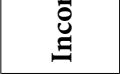 & 喅 & 鼠 & हี & 舀 & 舁 & 兒 & 鸹 & 鼠 & 鼠 & 声 & 告 & 声 & 告 \\
\hline 苞 & ह & 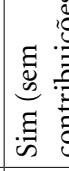 & 宩 & 总 & 总 & 舅 & 鼠 & 竧 & 鼠 & 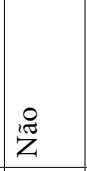 & 苟 & 鼠 & 鼠 \\
\hline 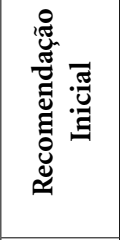 & 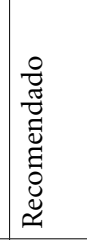 & 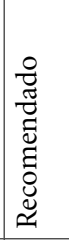 & 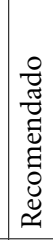 & 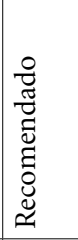 & 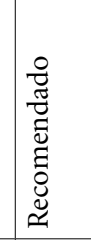 & 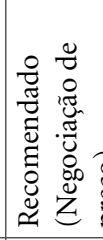 & 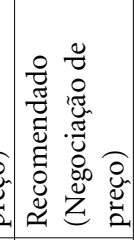 & 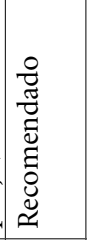 & 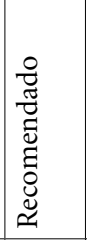 & 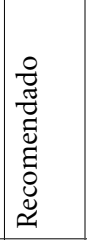 & 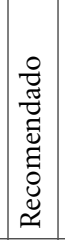 & 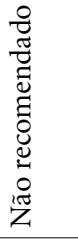 & 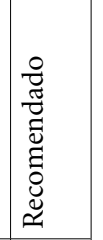 \\
\hline 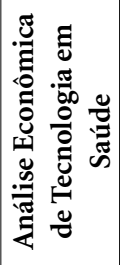 & 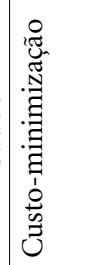 & 湂 & 湂 & 澏 & 兽 & 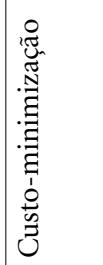 & 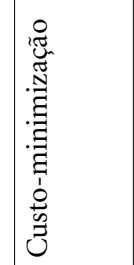 & 旁 & 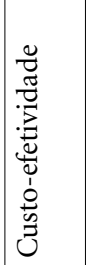 & 觉 & 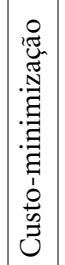 & : & 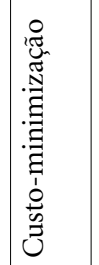 \\
\hline 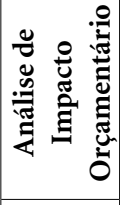 & ह & 罟 & 穵 & 湂 & 谞 & 兒 & 暍 & 授 & 慁 & 鼠 & 舁 & 鼠 & 兒 \\
\hline 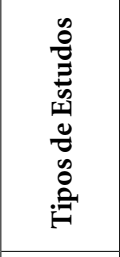 & 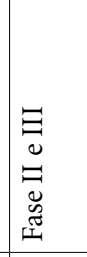 & 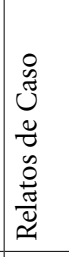 & 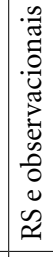 & 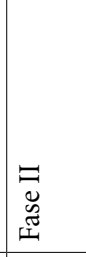 & 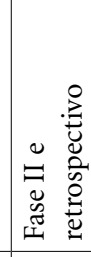 & 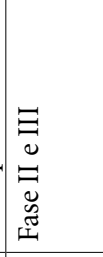 & 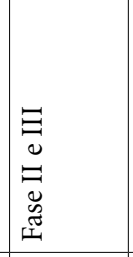 & 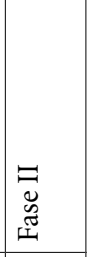 & 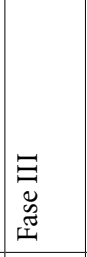 & 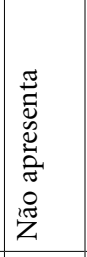 & 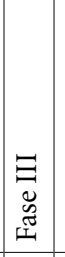 & 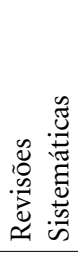 & 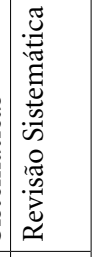 \\
\hline 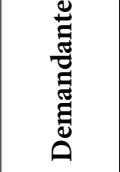 & $\begin{array}{l}\text { 㺃 } \\
\text { 鄫 }\end{array}$ & 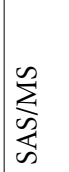 & $\sum_{\substack{n \\
5 \\
\vdots \\
\infty \\
\infty}}^{n}$ & $\sum_{\substack{n \\
\vdots \\
5 \\
\infty}}^{n}$ & 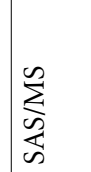 & 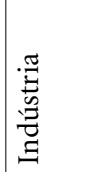 & 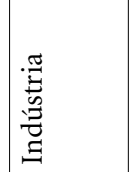 & 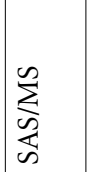 & 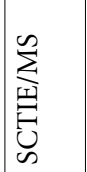 & 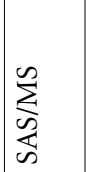 & 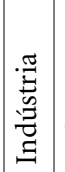 & 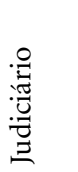 & 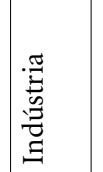 \\
\hline 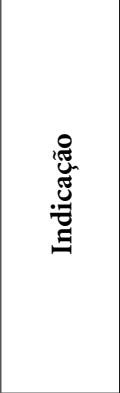 & 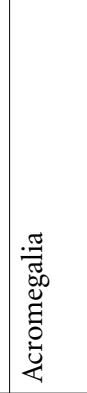 & 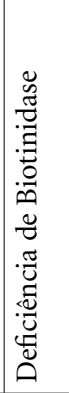 & 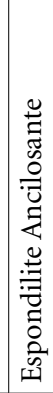 & 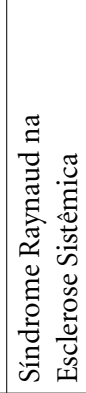 & 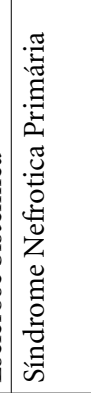 & 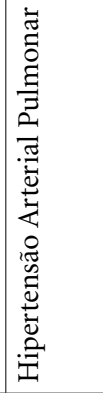 & 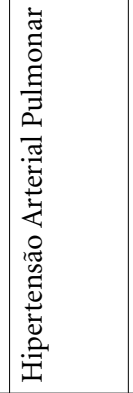 & 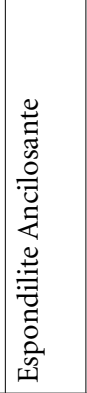 & 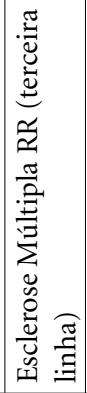 & 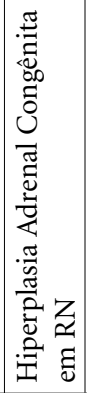 & 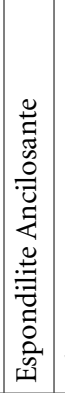 & 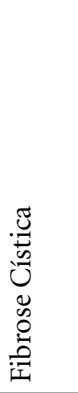 & 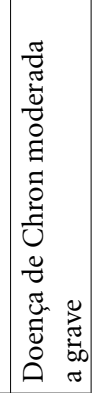 \\
\hline 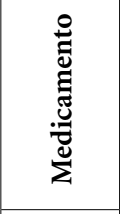 & 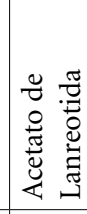 & & 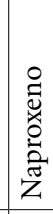 & 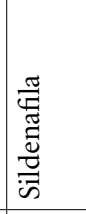 & 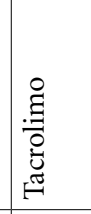 & 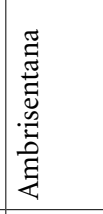 & 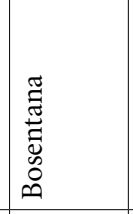 & 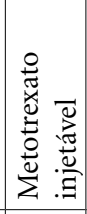 & 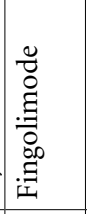 & 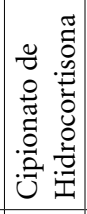 & 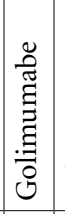 & 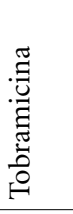 & 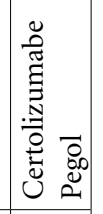 \\
\hline 章 & $\tilde{\stackrel{\sim}{~}}$ & तें & $\widetilde{d}$ & 亏ี & 亏ิ & 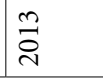 & ڤ్ন & $\stackrel{\sim}{\vec{\sim}}$ & ت্ّ & 旾 & 点 & 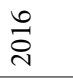 & 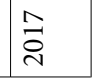 \\
\hline
\end{tabular}




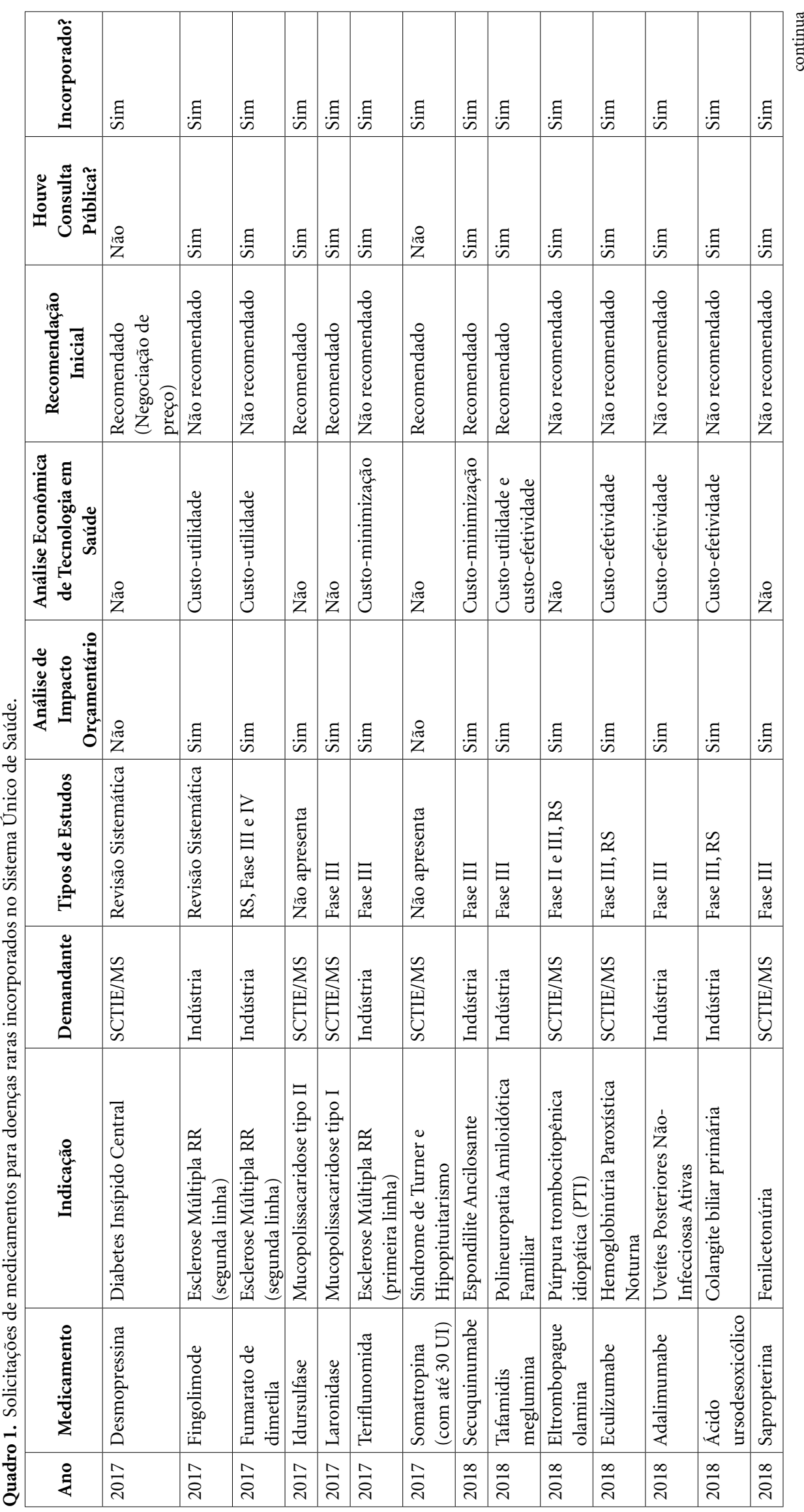


evidências clínicas, desde relatos de caso até revisões sistemáticas com metanálises. As revisões sistemáticas passaram a integrar os dossiês mais frequentemente a partir de 2017.

Análises de impacto orçamentário estavam presentes em cerca de $85 \%$ (51) das 60 solicitações enviadas à CONITEC, enquanto análises econômicas de tecnologias em saúde, tais como custo-minimização, custo-efetividade e custo-utilidade, estavam presentes em 68\% (41) delas. Como pode ser observado na Figura 1a, oito entre as nove solicitações que não apresentaram análise de impacto orçamentário tiveram a SAS/ MS, SCTIE/MS ou governos estaduais como demandantes. Com relação à falta de análises econômicas de tecnologias em saúde, 17 entre os 19 pedidos que não apresentaram qualquer análise deste tipo, também partiram dos mesmos demandantes supracitados (Figura 1b).

\section{As doenças raras demandadas}

Dentre as 30 doenças raras com solicitações de incorporação de medicamentos, esclerose múltipla teve o maior número de solicitações, doze ao total $(20 \%)$, sendo que apenas cinco destas foram incorporadas. Também podemos destacar a espondilite ancilosante [cinco solicitações (8\%), quatro incorporadas]; acromegalia [quatro solicitações (7\%), uma incorporada], hipertensão arterial pulmonar [quatro solicitações (7\%), duas incorporadas] e mucopolissacaridose [quatro solicitações (7\%), quatro incorporadas]. Os diferentes subtipos de cada doença não foram considerados ao agrupar o número de demandas por doença.

\section{Recomendações da CONITEC}

A Figura 2 mostra a evolução das solicitações de incorporação entre julho de 2012 e junho de 2019, bem como a caracterização dos demandantes quanto à recomendação inicial e à final da CONITEC. Das 61 avaliações de incorporação de medicamentos para doenças raras, apenas $20(33 \%)$ apresentaram recomendação inicial pré-consulta pública positiva. Entretanto, após consulta pública, houve uma inversão nestes números, e, desta forma, 32 (52\%) passaram a ter recomendação positiva, sendo de fato incorporadas ao SUS. Podemos notar que nos anos iniciais (2012 e 2013) houve um elevado número de solicitações enviadas à CONITEC, tanto por parte do governo quanto da indústria, e, todas as recomendações iniciais foram mantidas. As recomen- 


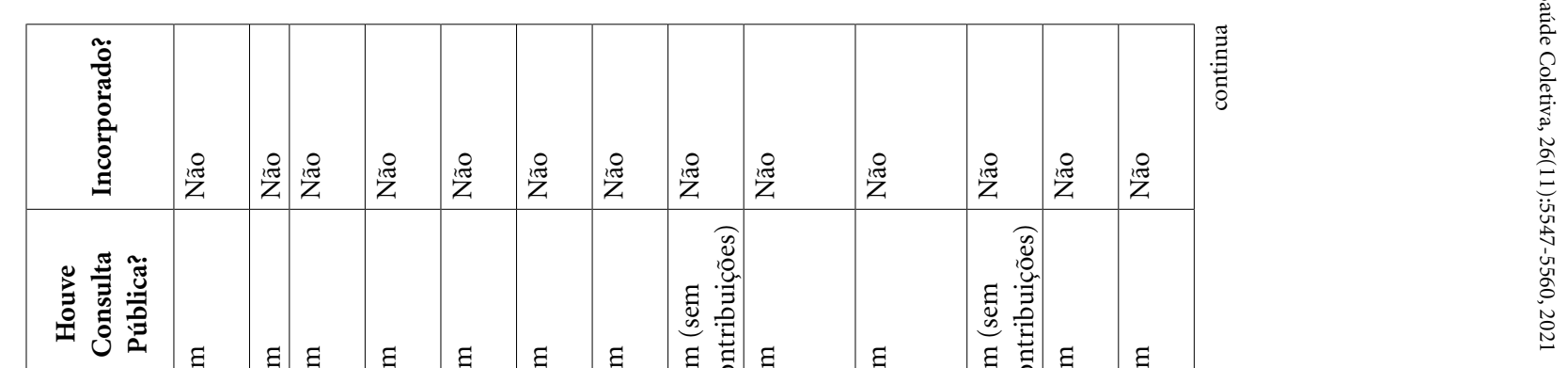

\begin{tabular}{|c|c|c|c|c|c|c|c|c|c|c|c|c|}
\hline 2 & है & 䍐鼠 & है & 灵 & 慁 & 灵 & है & हี & है & 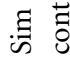 & : & क् \\
\hline 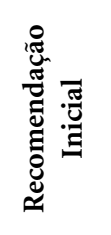 & 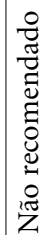 & 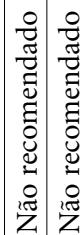 & 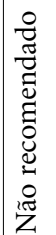 & 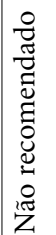 & 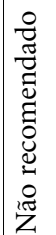 & 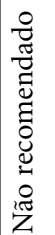 & 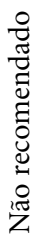 & 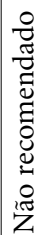 & 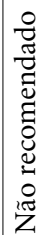 & 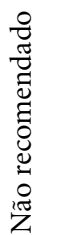 & 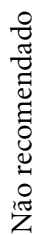 & 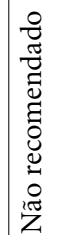 \\
\hline
\end{tabular}

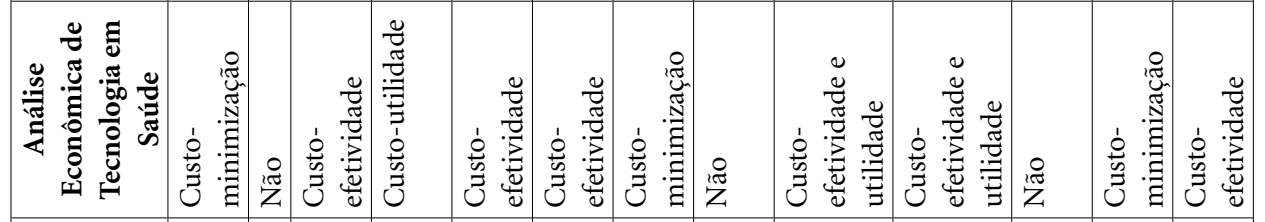

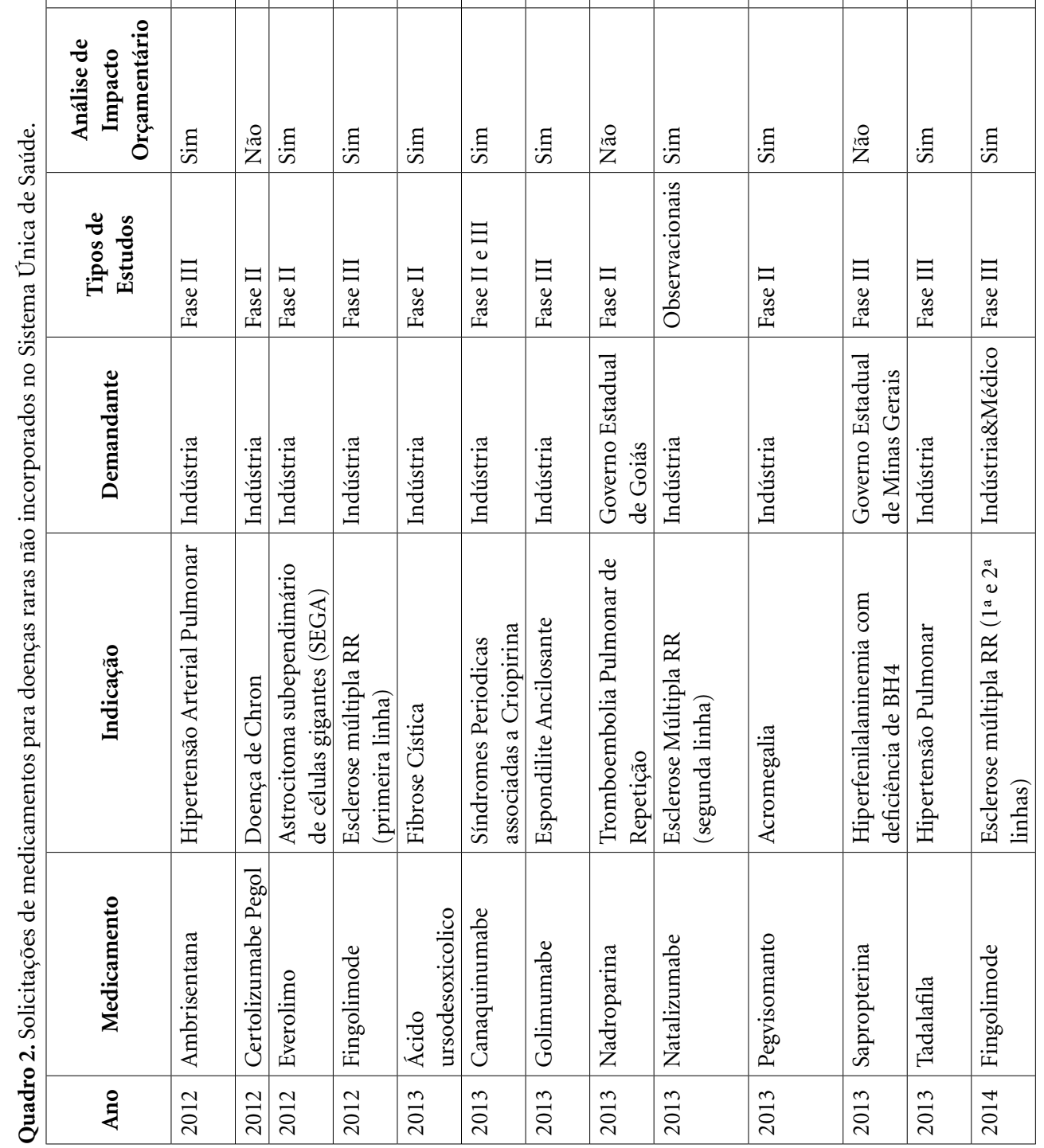




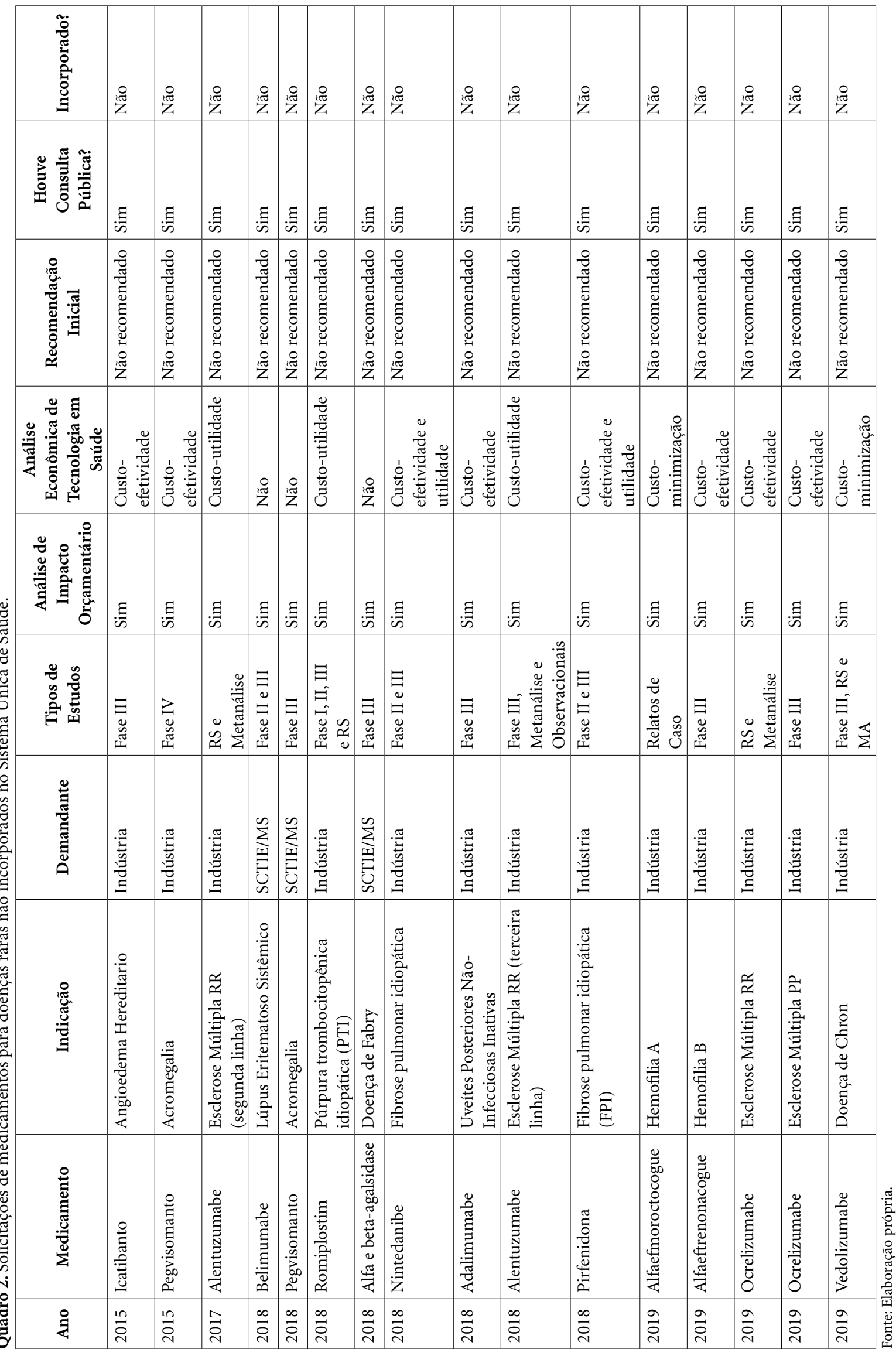



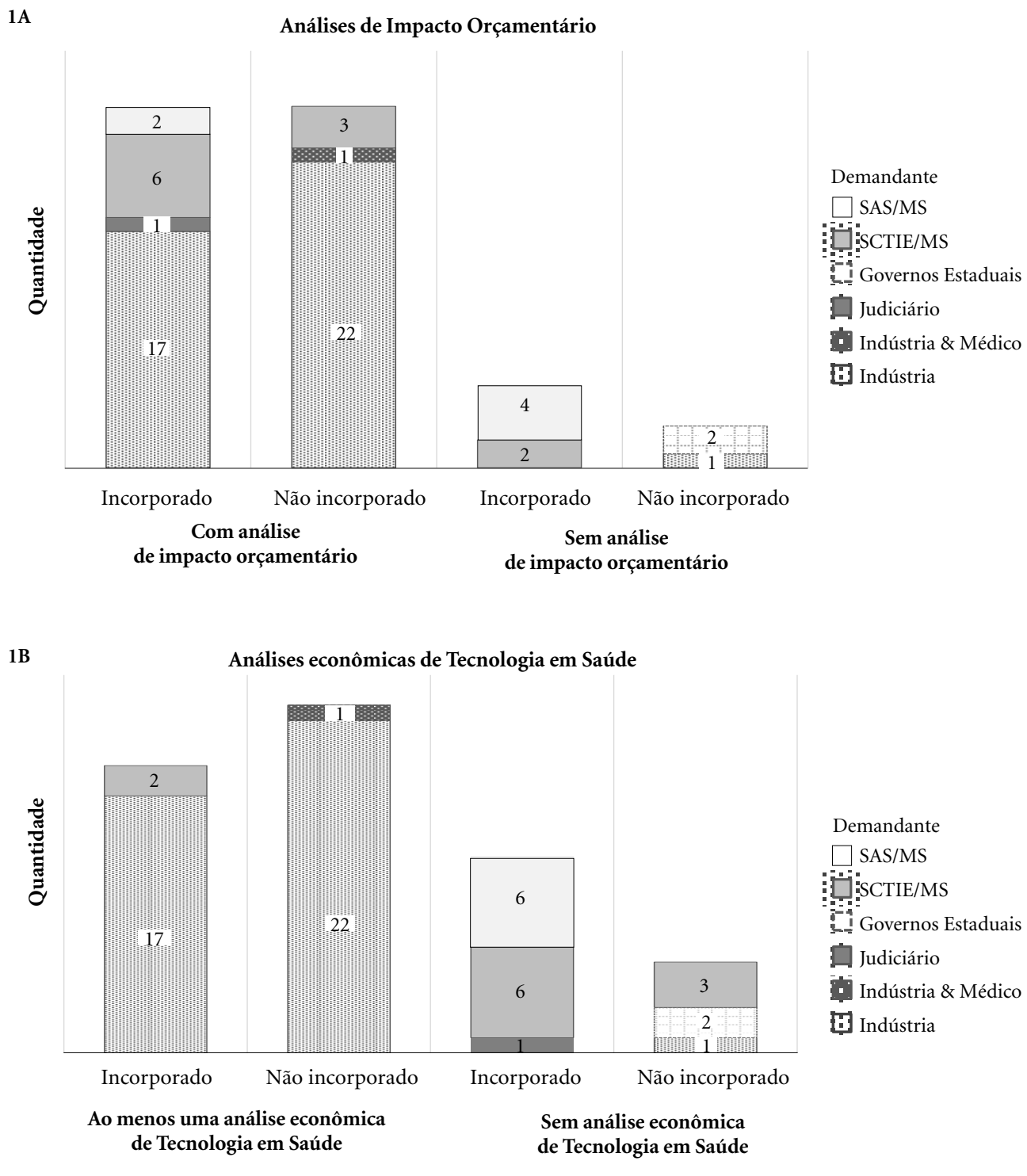

Figuras 1A e 1B. Quantidade de análises de impacto orçamentário e análises econômicas de tecnologias em saúde presentes nas solicitações incorporadas ou não pela CONITEC. SCTIE/MS: Secretaria de Ciência, Tecnologia e Insumos Estratégicos/Ministério da Saúde; SAS/MS: Secretaria de Atenção à Saúde/Ministério da Saúde.

Fonte: Elaboração própria a partir do levantamento dos pedidos de incorporação à CONITEC.

dações iniciais negativas começaram a ser revertidas a partir de 2016 e passaram a ser recorrentes nos anos seguintes, independentemente do tipo de demandante.

Das 12 solicitações de incorporação que tiveram decisões revertidas após consulta pública, as justificativas da CONITEC para a recomendação negativa inicial se apoiaram na ausência de evidências clínicas de qualidade, incerteza da efetividade, não serem opções custo-efetivas em relação às opções já incluídas no SUS, fragilidade das evidências e modestos benefícios clínicos que não justificam o alto preço. As evidências clínicas apresentadas pelos demandantes nestas solicitações foram, minimamente, estudos clínicos fase III.

As recomendações negativas iniciais que foram revertidas em positivas se apoiaram no alto número de contribuições favoráveis à incorporação de medicamentos para doenças raras; por 


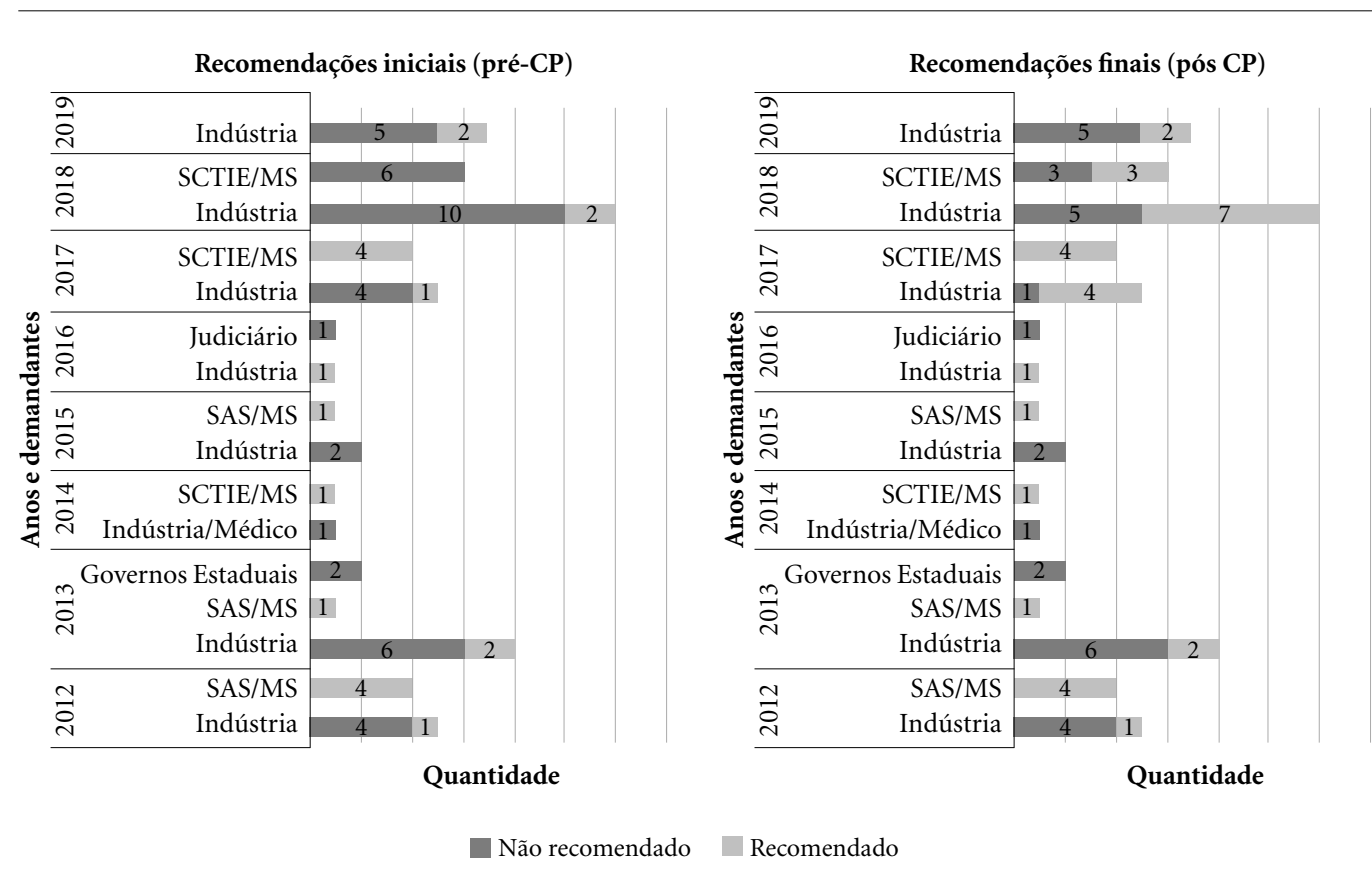

Figura 2. Evolução das recomendações iniciais (pré $\mathrm{CP}=$ pré consulta pública) e finais (pós $\mathrm{CP}=$ pós consulta pública) da CONITEC de julho de 2012 a junho de 2019. SCTIE/MS: Secretaria de Ciência, Tecnologia e Insumos Estratégicos/Ministério da Saúde; SAS/MS: Secretaria de Atenção à Saúde/Ministério da Saúde.

Fonte: Elaboração própria a partir do levantamento dos pedidos de incorporação à CONITEC.

exemplo, a Hemoglobinúria Paroxística Noturna que obteve 2.451 contribuições. As principais contribuições da consulta pública estavam relacionadas à apresentação de novos estudos clínicos, presença de medicamento genérico disponível no mercado, o medicamento solicitado era o único tratamento disponível para a doença, além da negociação de preço com a indústria.

\section{Critérios utilizados por agências de ATS internacionais para incorporação de medicamentos para doenças raras}

Um estudo realizado pela agência canadense $\mathrm{CADTH}^{19}$ comparou diversos processos de ATS para doenças raras em alguns países como Inglaterra, França, Austrália e o próprio Canadá. A Tabela 1 é uma adaptação de dados do relatório do CADTH e a coluna do Brasil foi respondida com base no levantamento bibliográfico feito para o desenvolvimento deste trabalho, apenas com dados públicos do próprio site da CONITEC.

Observa-se que apenas o NICE usufrui dos seis critérios adotados na avaliação, apesar de não definir a prevalência exata de uma doença considerada rara. Dentre as demais, apenas a PBAC possui um processo diferenciado para submissão de pedido de inclusão para doenças raras. Entre as quatro agências de ATS internacionais, todas usam como critério considerações diferenciadas quanto às avaliações econômicas. Vale destacar que apenas a Inglaterra e o Brasil informam estar dispostos a fazer acordos de partilha de risco com os demandantes das tecnologias.

\section{Discussão}

Diante dos dados apresentados neste estudo e tendo em vista que até o início das publicações das avaliações por parte da CONITEC a maioria dos medicamentos para doenças raras atendidas pelo SUS tratava apenas os sintomas ${ }^{20}$, pode-se notar importante avanço na forma como são tratados os pacientes com doenças raras. A maioria das tecnologias avaliadas entre julho de 2012 e junho de 2019 pela CONITEC incluiu medicamentos órfãos capazes de interferir na progressão da doença.

A Política Nacional de Atenção às Pessoas com Doenças Raras norteou caminhos e metas para que houvesse a priorização de alguns proto- 
Tabela 1. Critérios usados por agências de ATS internacionais para incorporação de medicamentos para doenças raras.

\begin{tabular}{|c|c|c|c|c|c|}
\hline \multirow{3}{*}{$\begin{array}{c}\text { Critérios usados pelas agências } \\
\text { de ATS }\end{array}$} & \multicolumn{5}{|c|}{ Países e suas agências de ATS } \\
\hline & Canadá & Inglaterra & França & Austrália & Brasil \\
\hline & CADTH & NICE & HAS & PBAC & CONITEC \\
\hline $\begin{array}{l}\text { Há um processo de ATS distinto para doenças } \\
\text { raras? Ou o processo padrão tem critérios } \\
\text { diferentes para doenças raras? }\end{array}$ & Não & Sim & Não & Sim & Não \\
\hline $\begin{array}{l}\text { Há critério de definição para os medicamentos } \\
\text { para doenças raras? }\end{array}$ & Não & $\operatorname{Sim}^{\star}$ & Não & Sim & Sim \\
\hline Há um comitê especial para auxiliar a avaliação? & Não & Sim & Não & Não & Não \\
\hline $\begin{array}{l}\text { Pacientes ou grupos de pacientes fazem parte do } \\
\text { comitê de avaliação especial? }\end{array}$ & NR & Sim & NR & NR & Não \\
\hline $\begin{array}{l}\text { Alguma consideração diferenciada é feita para } \\
\text { avaliação econômica? }\end{array}$ & Sim & Sim & Sim & Sim & Não \\
\hline Acordos de risco compartilhado são considerados? & NR & Sim & NR & NR & Sim \\
\hline
\end{tabular}

${ }^{\star}$ Apesar de ter o critério de ser doença rara, não existe prevalência bem definida para sua definição. NR: Não reportado.

Fonte: Elaboração própria a partir do levantamento dos critérios das agências internacionais.

colos clínicos e diretrizes terapêuticas (PCDT) $)^{13}$. A partir da necessidade de acelerar a chegada dos medicamentos órfãos criada após essa Política de $2014^{13}$, a ANVISA, com a RDC 205/17, estipulou um procedimento para registro de medicamentos órfãos: os medicamentos registrados por meio de critérios pré-estabelecidos, seriam priorizados tendo o prazo de até 365 dias para serem comercializados $^{21}$. Aos poucos, então, toma forma a maneira como o sistema de saúde brasileiro lida com doenças raras. É possível que, entre outros fatores, haja uma relação causal entre o aumento do número de demandas de incorporação a partir de 2017 e este novo cenário para doenças raras, tal como ocorreu nos EUA após instauração de incentivos e legislações (Orphan Drug Act) $)^{22,23}$.

Com os avanços por meio de Políticas e Resoluções, os pacientes passaram a ter a chance de serem tratados pelo sistema de saúde de maneira mais digna. Tem-se então, a tentativa de fazer valer dois dos três princípios do SUS ${ }^{1}$ : a equidade, já que pacientes que sofrem de condições raras devem ter a mesma oportunidade de receber tratamento que outros pacientes com distúrbios mais frequentes ${ }^{22}$; e a universalidade, por meio da inclusão deste grupo de pacientes nas políticas públicas de saúde. Já a integralidade, devido à dificuldade de organizar um sistema que consiga cuidar do paciente em sua totalidade, seja em doenças raras ou mais prevalentes ${ }^{24}$, ainda é um dos aspectos almejados.

É importante destacar que, apesar da maioria das solicitações de incorporação enviadas à CONITEC ter tido decisão positiva, o acesso ao tratamento pode ser mais difícil do que se espera. Problemas de logística, distribuição e abastecimento são frequentes nas farmácias do componente especializado, gerando impacto na disponibilidade e, portanto, no tratamento dos usuários de medicamentos ${ }^{25}$.

A questão fundamental quando se discute o presente tema é o impacto orçamentário que tais incorporações acarretam ao sistema, já que se trata de medicamentos para um número restrito de pacientes e geralmente têm alto custo de fabricação e venda. De acordo com estimativas do Ministério da Saúde publicadas pela Interfarma ${ }^{20}$, somente em 2016, os gastos da União com ações judiciais atingiram $\mathrm{R} \$ 1,3$ bilhão, um aumento de $23 \%$ em relação ao ano anterior. A participação dos medicamentos órfãos nestes gastos por via judicial já representa $90 \%$ do custo total, com metade dos medicamentos mais judicializados em 2016 sendo para tratamento de doenças raras ${ }^{20}$. Desta forma, já existe um alto gasto com aquisição de medicamentos para esse grupo de doenças por parte da União, somado à falta de previsibilidade na alocação de recursos, diminuição no poder de negociação com as indústrias e menor número de pacientes beneficiados. Ante este cenário, é possível que a incorporação destes medicamentos no SUS possa trazer maior controle nos gastos com ações judiciais e ainda possibilitar tratamento a um maior número de pacientes.

A comparação dos critérios utilizados por agências internacionais de ATS explicita que a 
forma como é avaliada uma doença rara é um tema atual e ainda precisa de amadurecimento. Entende-se que, por mais que o país invista em saúde, não é possível pagar tudo para todos ${ }^{7}$. Por esse motivo, debater formas mais efetivas, além da metodologia de custo-efetividade e impacto orçamentário, para avaliar as novas tecnologias se faz tão importante. Como se observou nas cinco agências de ATS incluídas neste trabalho, critérios diferenciados (utilizando princípios menos utilitaristas e considerando tanto a vulnerabilidade da população atingida quanto à posição da sociedade em relação a essa inclusão) e com definição de prioridades, pode ser a forma mais indicada para avaliar os medicamentos para doenças $\operatorname{raras}^{8} \mathrm{e}$, assim, melhorar o acesso a medicamentos a essa população tão diferenciada. Além disso, alguns novos programas específicos para avaliar medicamentos para doenças raras vêm surgindo na Europa como tentativa de enfrentar o cenário desafiador de maior pressão política por mais transparência nos processos de ATS $^{26}$, como o Patient and Clinician Engagement (PACE) Meeting e o programa de tomada de decisão do Scottish Medicines Consortium (SMC), o programa para tecnologia altamente especializada do National Institute for Health and Clinical Excellence (NICE) na Inglaterra e uma iniciativa europeia, o Mechanism of Coordinated Access $(\mathrm{MoCA})^{27}$.

Entendendo a integralidade como princípio que permeia as tomadas de decisão e a garantia do direito à saúde, emergem alguns questionamentos cruciais: as incorporações de medicamentos se realizam a partir de critérios que levem em consideração as necessidades em saúde e a perspectiva da integralidade? É possível atenção integral aos pacientes com doenças raras usando os atuais critérios de avaliação de tecnologias em saúde? Um caminho inicial - ainda que complexo - para tomadores de decisão, poderia ser a identificação dos fatores de valor que julgam relevantes para cada decisão, as preferências dos grupos envolvidos, quais proposições de valor sustentam as decisões e a construção de um consenso entre todas as partes ${ }^{26,28,29}$. Sendo assim, é preciso tornar o processo de trabalho mais transparente e participativo, só assim poder-se-ia construir decisões coletivas levando em consideração perspectivas minoritárias e até mesmo individuais, sem com- prometer grupos majoritários. Isto possibilitaria a diferenciação dos critérios ainda hoje muito utilizados igualmente tanto para doenças de alta quanto de baixa prevalência.

A própria CONITEC, em três das 12 solicitações em que houve inversão na recomendação inicial, justificou a recomendação final positiva após consulta pública da seguinte maneira: as evidências científicas em relação às doenças raras devem ser analisadas de modo diferente quando comparadas às doenças de alta prevalência. Da mesma forma, verificou-se a necessidade de acompanhamento destes pacientes para que os resultados do tratamento sejam monitorados e documentados, presente nos relatórios da Mucopolissacaridose IVa e $\mathrm{VI}^{30,31}$. Além disso, reconsiderou-se a decisão mediante o cumprimento de critérios provisoriamente estabelecidos para doenças raras - relatório de Hemoglobinúria Paroxística Noturna ${ }^{32}$.

\section{Conclusão}

Desde a criação da CONITEC, o cenário de doenças raras mudou no sistema público de saúde brasileiro. Não podemos negar os avanços conquistados que já foram observados a partir dos dados apresentados neste trabalho, em que a maioria (52\%) dos medicamentos que a CONITEC avaliou nos últimos sete anos foram incorporados ao SUS. Entretanto, ainda há desafios e oportunidades não só no Brasil, mas também em países cujas agências de ATS foram pioneiras, tamanha a relevância e atualidade do tema. Países como Inglaterra e a Austrália, por exemplo, já apresentam critérios diferenciados significativos em suas agências de ATS.

Por outro lado, os recursos limitados na saúde e o cenário político econômico são pontos de atenção e dificuldade que enfrentamos no País. Entretanto, para que os avanços não sejam desacelerados, é importante trazer para a luz da consciência como estão se dando os gastos da União, sobretudo, com judicializações. Este ponto, associado a critérios diferenciados e fatores de valor relevantes para doenças raras, além de políticas com objetivos bem definidos, possivelmente trará robustez à tomada de decisão e maior potencial de acesso dos pacientes a tratamentos que podem mudar suas vidas. 


\section{Colaboradores}

LV Biglia e PM Aguiar participaram da concepção do projeto, análise e interpretação dos dados, redação do artigo e revisão crítica do conteúdo intelectual. SJ Mendes e TM Lima colaboraram na discussão dos dados e na revisão crítica do texto.

\section{Referências}

1. Brasil. Lei 8080 de 19 de setembro de 1990. Dispõe sobre as condições para a promoção, proteção e recuperação da saúde, a organização e o funcionamento dos serviços correspondentes e dá outras providências. Diário Oficial da União 1990; set 20.

2. Brasil. Constituição da República Federativa do Brasil. Diário Oficial [da] República Federativa do Brasil, Brasília, DF, 1988. [acessado 2020 jan 18]. Disponível em: http://www.planalto.gov.br/ccivil_03/constituicao /constituicaocompilado.htm.

3. Brasil. Ministério da Saúde (MS). Secretaria de Ciência, Tecnologia e Insumos Estratégicos. Departamento de Ciência e Tecnologia. Política Nacional de Gestão de Tecnologias em Saúde. Brasília: MS; 2010. 47 p.

4. Carnut L. Cuidado, integralidade e atenção primária: articulação essencial para refletir sobre o setor saúde no Brasil. Saude Debate 2017; 41(115):1177-1186.

5. Brasil. Ministério da Saúde (MS). Portaria GM/MS no 3916 de 30 de outubro de 1998. Aprova a Política Nacional de Medicamentos. Brasília, DF. Diário Oficial da União, Poder Executivo, Brasília, DF, 10 de novembro de 1998.

6. Brasil. Conselho Nacional de Saúde. Resolução no 338 de 6 de maio de 2004. Aprova a Política Nacional de Assistência Farmacêutica. Diário Oficial da União, Poder Executivo, Brasília, DF, 20 de maio de 2004.

7. Soárez PC, Soares MO, Novaes HMD. Modelos de decisão para avaliações econômicas de tecnologias em saúde. Cien Saude Colet 2014; 19(10):4209-4222.

8. Brasil. Lei No 12.401 , de 28 de abril de 2011.Altera a Lei no 8.080, de 19 de setembro de 1990, para dispor sobre a assistência terapêutica e a incorporação de tecnologia em saúde no âmbito do Sistema Único de Saúde - SUS. Diário Oficial da União, Brasília, DF, 29 de abril de 2011.

9. Silva EN, Sousa TRV. Avaliação econômica no âmbito das doenças raras: isto é possível? Cad Saude Publica 2015; 31(3):1-11.

10. Remuzzi G, Garattini S. Rare diseases: what's next? Lancet 2008; 371(9629):1978-1979.

11. Schieppati A, Henter JI, Daina E, Aperia A. Why rare diseases are an important medical and social issue. Lancet 2008; 371(9629):2039-2041.

12. World Health Organization (WHO). Bulletin of the World Health Organization. Coming together to combat rare diseases. Vol. 90, Number 6, June 2012, 401476.

13. Brasil. Ministério da Saúde (MS). Política Nacional de Atenção Integral às Pessoas com Doenças Raras. Portaria GM no 199, de 30 de janeiro de 2014. [acessado 2020 jan 18]. Disponível em: http://bvsms.saude.gov. br/bvs/saudelegis/gm/2014/prt0199_30_01_2014. html.

14. Auvin S, Irwin J, Abi-Aad P, Battersby A. The Problem of Rarity: Estimation of Prevalence in Rare Disease. Value Health 2018; 21(5):501-507.

15. Brasil. Ministério da Saúde (MS). Priorização de Protocolos e Diretrizes Terapêuticas para Atenção Integral às Pessoas com Doenças Raras. No $142 \mathrm{Maio} / 2015$.

16. Aith FMA. O direito à saúde e a política nacional de atenção integral aos portadores de doenças raras no Brasil. JBES 2014; 6(Supl.1):4-12. 
17. Sartori Junior D, Leivas PGC, Souza MV, Krug BC, Balbinotto G, Schwartz IVD. Judicialização do acesso ao tratamento de doenças genéticas raras: a doença de Fabry no Rio Grande do Sul. Cien Saude Colet 2012; 17(10):2717-2728.

18. Guimarães R. Incorporação tecnológica no SUS: o problema e seus desafios. Cien Saude Colet 2014; 19(12):4899-4908.

19. Pant S, Visintini S. Drugs for rare diseases: a review of national and international health technology assessment agencies and public payers' decision-making processes. Ottawa: CADTH; 2018. (Environmental scan; $\mathrm{n}^{\circ}$ 77). [acessado 2019 abr 14]. Disponível em: https:// www.cadth.ca/recommendations-framework-drugs -rare-diseases-review-national-and-international-health-technology

20. Associação da Indústria Farmacêutica de Pesquisa (Interfarma). Doenças Raras: A urgência do acesso à saúde. São Paulo: Interfarma; 2018. [acessado 2019 abr 14]. Disponível em: https://www.interfarma.org. br/public/files/biblioteca/doencas-raras--a-urgenciado-acesso-a-saude-interfarma.pdf.

21. Brasil. Agência Nacional de Vigilância Sanitária (Anvisa). Resolução da Diretoria Colegiada - $n^{\circ} 205$, de 28 de dezembro de 2017. Procedimento especial para anuência de ensaios clínicos, certificação de boas práticas de fabricação e registro de novos medicamentos para tratamento, diagnóstico ou prevenção de doenças raras. Brasília: Anvisa; 2019. [acessado 2019 abr 14]. Disponível em: http://portal.anvisa.gov.br/documents/ 10181/2718376/RDC_205_2017_.pdf/996fc46e-216b -44ab-b8c8-2778151b786e.

22. Drummond MF, Wilson D, Kanavos P, UbelP. Assessing the economic challenges posed by orphan drugs. Int J Technol Assess Health Care 2007; 23(1):36-42.

23. US Food and Drug Administration. Orphan Drug Act of 1983. Pub L. No 97-414, 96 Stat. 2049. 4 January 1983. Retrieved 27 October 2015. [acessado 2019 abr 14]. Disponível em: https://www.govinfo.gov/content/pkg/STATUTE-96/pdf/STATUTE-96-Pg2049. pdf.

24. Aith F, Bujdoso Y, Nascimento PR, Dallari S. Os princípios da universalidade e integralidade do SUS sob a perspectiva da política de doenças raras e da incorporação tecnológica. $R$ Dir Sanit 2014; 15(1):10-19.

25. Blatt CR, Campos CMT, Becker IRT.Assistência Farmacêutica no Brasil: Política, Gestão e Clínica. Logística de medicamentos. Cap. 3, Vol. IV, Santa Catarina: Editora UFSC; 2016.

26. Novaes HMD, Soárez PC. Doenças raras, drogas órfãs e as políticas para avaliação e incorporação de tecnologias nos sistemas de saúde. Sociologias 2019; 21(51):332-364.
27. Nicod E, Annemans L, Bucsics A, Lee A, Upadhyaya S, Facey K. HTA programme response to the challenges of dealing with orphan medicinal products: process evaluation in selected European countries. Health Policy 2019; 123(2):140-151.

28. Paulden M, Stafinski T, Menon D, McCabe C. Value -based reimbursement decisions for orphan drugs: a scoping review and decision framework. Pharmacoeconomics 2015; 33(3):255-269.

29. BaickerK, Chandra A. Evidence-based health policy. $N$ Engl J Med 2017; 377:2413-2415.

30. Comissão Nacional de Incorporação de Tecnologias no SUS (CONITEC). Relatório 412- Galsulfase para a terapia de reposição enzimática de longo prazo, em pacientes com diagnóstico confirmado de mucopolissacaridose tipo VI (deficiência de N-acetilgalactosamina 4-sulfa). 2018. [acessado 2019 abr 20]. Disponível em: http://conitec.gov.br/images/Relatorios/2018/Recomendacao/Relatorio_Galsulfase_MPS_VI.pdf.

31. Comissão Nacional de Incorporação de Tecnologias no SUS (CONITEC). Relatório 411 - Alfaelosulfase para pacientes com mucopolissacaridose tipo IVa (Síndrome de Morquio A). 2018. [acessado 2019 abr 20]. Disponível em: http://conitec.gov.br/images/Relatorios/2018/Relatorio_Alfaelosulfase_MPS_IVa.pdf.

32. Comissão Nacional de Incorporação de Tecnologias no SUS (CONITEC). Relatório $n^{\circ} 413$ - Eculizumabe para o tratamento da Hemoglobinúria Paroxística Noturna. 2018. [acessado 2019 abr 20]. Disponível em: http://conitec.gov.br/images/Relatorios/2018/Relatorio_Eculizumabe_HPN.pdf.

Artigo apresentado em 02/05/2020

Aprovado em 27/07/2020

Versão final apresentada em 29/07/2020

Editores-chefes: Romeu Gomes, Antônio Augusto Moura da Silva 